\title{
Probing Surface Properties of Cytochrome $c$ at Au Bionanoconjugates
}

\author{
Inês Gomes, ${ }^{\dagger}$ Nuno C. Santos, ${ }^{\ddagger}$ Luís M. A. Oliveira, ${ }^{\S}$ Alexandre Quintas, ${ }^{\S}$ Peter Eaton,, \\ Eulália Pereira," and Ricardo Franco*,† \\ REQUIMTE, Departamento de Química, Faculdade de Ciências e Tecnologia, Universidade Nova de Lisboa, \\ 2829-516 Caparica, Portugal, Instituto de Medicina Molecular, Faculdade de Medicina da Universidade de \\ Lisboa, Av. Prof. Egas Moniz, 1649-028 Lisboa, Portugal, Laboratório de Patologia Molecular, Instituto \\ Superior de Ciências da Saúde Egas Moniz, Campus Universitário, Quinta da Granja, 2829-511 Caparica, \\ Portugal, and REQUIMTE/Faculdade de Ciências, Universidade do Porto, 4169-007 Porto, Portugal
}

Received: May 29, 2008; Revised Manuscript Received: July 19, 2008

\begin{abstract}
Bionanoconjugates were created with cytochrome $c$ from horse heart (HCc) or yeast (YCc) and citrate-stabilized gold nanoparticles (AuNPs). Evidence for the formation of stable $\mathrm{HCc}-\mathrm{AuNP}$ and $\mathrm{YCc}-\mathrm{AuNP}$ bionanoconjugates came from a $5 \mathrm{~nm}$ red-shift of the surface plasmon resonance band of the AuNPs, increase of the $\zeta$-potential, and direct visualization by atomic force microscopy. Langmuir isotherm fittings of $\zeta$-potential data indicated that higher enthalpy changes are involved in the formation of the HCc-AuNP than in YCc-AuNP. UV-vis and circular dichroism studies of $\mathrm{pH}$-induced aggregation of the bionanoconjugates revealed distinct protonation patterns with an aggregation $\mathrm{pH}$ of 8.8 and 6.2 for $\mathrm{YCc}-\mathrm{AuNP}$ and $\mathrm{HCc}-\mathrm{AuNP}$, respectively. No appreciable changes were observed in the secondary structure of $\mathrm{HCc}$ in $\mathrm{HCc}-\mathrm{AuNP}$. In contrast, YCc in YCc-AuNP presented a decrease in $\alpha$-helix content upon AuNP binding and an increase in $\beta$-sheet content upon $\mathrm{pH}$-induced aggregation. Data discussion is based on the distinct binding modes of both proteins to the AuNPs via a covalent bond (Cys 102) for YCc and via electrostatic interaction for HCc.
\end{abstract}

\section{Introduction}

Gold nanoparticles (AuNPs) present unique optical properties that make them especially suited for application in bioassays and cell targeting. ${ }^{1-3}$ Conjugation of proteins with AuNP either directly ${ }^{4-8}$ or via tailored ligands forming a capping monolayer ${ }^{9-13}$ affords bionanoconjugates that are especially adequate for biological interactions. ${ }^{2,14,15}$ Characterization of the physical processes involved in protein interaction with the AuNPs and the stability and robustness of the resulting bionanoconjugates is of critical importance in this context. ${ }^{13,16}$

Typically, a solution of spherical AuNPs is red due to the collective oscillation of electrons in the conduction band known as the surface plasmon resonance (SPR). ${ }^{17}$ This band presents an exceptionally high absorption coefficient, centered at ca. 520 $\mathrm{nm}$. The wavelength of the SPR depends weakly on the size of the particle or the refractive index of the surrounding media ${ }^{18-20}$ but strongly changes with interparticle distance. ${ }^{21}$ Color change of the solution can then be induced by AuNP aggregation, a phenomenon that has been advantageously utilized in specific DNA sequence detection for biomedical applications, $, 3,22,23$ in determination of enzymatic activity, ${ }^{24-26}$ or as a probe for protein conformational changes. ${ }^{5,27}$

Cytochrome $c$ (Cyt $c$ ) is a low molecular mass protein (ca. $12 \mathrm{kDa}$ ) containing a heme group. The " $c$ " denomination implies that the heme group is covalently bound to two cysteines of the amino acid sequence, in opposition to hemes of the " $b$ " type like the ones found in myo- and hemoglobin in which the heme is noncovalently attached to the protein. Cytochrome $c$ is easy

* To whom correspondence should be addressed. E-mail: r.franco@ dq.fct.unl.pt. Telephone: +351-212 949 659. Fax: +351-212 948550.

$\dagger$ Universidade Nova de Lisboa.

* Faculdade de Medicina da Universidade de Lisboa.

§ Instituto Superior de Ciências da Saúde Egas Moniz.

"Universidade do Porto. to obtain in a pure form and remains stable under various solution conditions, withstanding $\mathrm{pH}$, ionic strength, and temperature variations without denaturation or otherwise loosing its native three-dimensional and active structure. ${ }^{28}$ Cytochrome $c$ derived from horse heart ( $\mathrm{HCc}$ ) has been extensively used as a model protein because it acts as an electron carrier in the respiratory chain of a mammal and plays an important role in programmed cell death. ${ }^{29,30}$ Yeast iso-1-cytochrome $c$ isolated from Saccharomyces cerevisiae (YCc) shares a highly homologous amino acid sequence with $\mathrm{HCc}(80 \%$ similarity as determined by the CLUSTALW Alignment Software ${ }^{31}$ ), and the three-dimensional structures from both proteins are practically superimposable. ${ }^{32}$ Nevertheless, YCc can not replace HCc in certain cell regulatory aspects, like caspase activation. ${ }^{30}$ Both proteins present a "front face lysine patch" in their threedimensional structures, a protein region that is positively charged at neutral $\mathrm{pH}$ and is involved in the formation of physiological complexes of cytochromes $c$ with other proteins. ${ }^{33,34}$ This highly charged region has been advantageously utilized for studying adsorption to negatively charged surfaces in, for example, electrochemical systems. ${ }^{35}$ Approximately opposite to the "lysine patch", YCc features Cys102 located next to the surface-exposed C-terminal Glu and structurally close to the exposed heme edge. ${ }^{36}$ This free cysteine on the surface has been advantageously used for specific tethering, ensuring unique orientation of the protein, and generating electrode-immobilized YCc films with great potential in bioelectronic applications. ${ }^{37,38}$ The physicochemical properties of these films, such as their $\mathrm{pH}-$ dependent equilibria, ${ }^{39}$ and electron transfer properties in context with topological analysis ${ }^{38,40}$ have been explored. Conversely, HCc lacks a free Cys residue implying that its interaction with metal surfaces and electrodes cannot be established via such a covalent attachment. 
Protein binding to AuNPs, as a means to form bionanoparticles for bioassays and protein therapy, has been addressed from the point of view of maintaining the protein functionality and activity, avoiding structural changes on the protein. In this context, monolayer-protected nanoparticles appear quite promising, featuring metal, semiconductor, and magnetic cores with useful optical, electronic, and magnetic properties and surface properties that can be tailored by selection of the appropriated capping ligands. This approach has led to novel biosensors, ${ }^{15}$ diagnostic agents, ${ }^{14}$ and nanostructured materials. ${ }^{41}$

The interaction of proteins with citrate-stabilized AuNP constitutes a more direct protein-AuNP interaction as citrate is a rather labile capping ligand known to be easily replaced by other stronger binding ligands such as thiolates or amine and carboxylates; these are functionalities that are ubiquitous in protein primary structures. In this work, we describe the formation and surface characterization of bionanoconjugates formed by an AuNP core and adsorbed cytochromes $c$. Two types of cytochromes $c$ featuring two different binding modes to the AuNPs were used: YCc that binds via a $-\mathrm{S}$ covalent bond and $\mathrm{HCc}$ that shows an electrostatic interaction with the AuNPs. The formation of the bionanoconjugates was evaluated by dynamic light scattering and $\zeta$-potential measurements as well as direct visualization by atomic force microscopy (AFM). The different modes of binding of the proteins to the AuNPs impaired different surface properties to the bionanoconjugates, namely at the levels of its $\mathrm{pH}$ induced aggregation behavior as evaluated by UV-vis spectroscopy and the protein secondary structure as assessed by circular dichroism (CD) spectroscopy.

\section{Experimental Section}

Chemicals. Horse heart cytochrome $c$ and Yeast-iso-1cytochrome $c$ from Saccharomyces cerevisiae were purchased from Sigma-Aldrich and prepared in $20 \mathrm{mM}$ potassium phosphate buffer, $\mathrm{pH}$ 7.2. The concentration of cytochrome $c$ was determined spectrophotometrically using a molar absorptivity of $29.5 \mathrm{mM}^{-1} \mathrm{~cm}^{-1}$ at $551 \mathrm{~nm}$.

All chemicals were from Sigma-Aldrich or Riedel-de-Haën and of the highest purity available.

Gold Nanoparticles Synthesis and Preparation of Cyt c-AuNP Bionanoconjugates. Gold nanoparticles (AuNPs) were synthesized by the citrate reduction method as previously described. ${ }^{42}$ Cytochrome $c$-AuNP conjugates (bionanoconjugates) were prepared by overnight incubation of an as-prepared AuNP solution with $\mathrm{pH}$ adjusted to 11.0 by addition of $0.1 \mathrm{M}$ $\mathrm{NaOH}$, with an appropriate amount of a Cyt $c$ solution to obtain the Cyt $c$-AuNP bionanoconjugates with the desired [Cyt $c] /[\mathrm{AuNP}]$ ratio. Heart cytochrome $c(\mathrm{HCc})$ and Yeast-iso-1cytochrome $c$ (YCc) were utilized to prepare AuNP- HCc and AuNP-YCc bionanoconjugates, respectively.

Microscopy and Spectroscopy Measurements. To prepare the AFM samples of AuNPs alone or bionanoconjugates, samples were centrifuged for $25 \mathrm{~min}$ at $30000 \mathrm{~g}$, followed by removal of supernatant. The precipitate was washed twice with $100 \mu \mathrm{L}$ of molecular biology grade water (Sigma-Aldrich) and finally resuspended in $60 \mu \mathrm{L}$ of water to discard salt/buffer crystals that would affect AFM measurements. Samples were then pipetted onto freshly cleaved mica followed by drying. Imaging was carried out with a Veeco Multimode AFM with a Nanoscope 4a controller. The AFM was operated in tapping mode, and rectangular silicon cantilevers of resonant frequency about 300 kHz (AppNano, CA) were used.

Absorption spectra were performed in a UNICAM, model UV2, UV-vis spectrophotometer. Quartz cells were from
Hellma, Germany and were at room temperature. The $\mathrm{pH}$ value of each bionanoconjugate sample was adjusted by the addition of a $0.1 \mathrm{M} \mathrm{HCl}$ solution, and spectra or photographs were taken immediately afterward.

CD spectra were collected on a Jasco-810 Spectropolarimeter, equipped with a thermoelectric temperature control. The $\mathrm{pH}$ of the bionanoconjugate samples was adjusted with a $0.1 \mathrm{M}$ phosphoric acid solution. Spectra were measured in a quartz cuvette with a $1 \mathrm{~mm}$ path length from 195 to $260 \mathrm{~nm}$ at a rate of $20 \mathrm{~nm} \mathrm{~min}{ }^{-1}$ with a sampling interval of $0.1 \mathrm{~nm}$ and $16 \mathrm{~s}$ response time. The temperature was held constant at $25{ }^{\circ} \mathrm{C}$. Analyses of the CD spectra were performed online using DICHROWEB (www.cryst.bbk.ac.uk/cdweb/html/home.html) with the CDSSTR algorithm. ${ }^{43,44}$ Results were expressed as mean residue ellipticity, $[\theta]$, in $\operatorname{deg~} \mathrm{cm}^{2} \mathrm{dmol}^{-1}$ at $222 \mathrm{~nm}$.

Light-Scattering Measurements. Dynamic light scattering (DLS) and $\zeta$-potential measurements were performed in a Zetasizer Nano-ZS from Malvern Instruments. A $4 \mathrm{~mW} \mathrm{He}-\mathrm{Ne}$ laser $(633 \mathrm{~nm})$ was used with a fixed 173 (DLS) or $17^{\circ}(\zeta$ potential) scattering angle. All measurements were carried out at $25{ }^{\circ} \mathrm{C}$. One milliliter of the sample at $\mathrm{pH} 11$ previously prepared in a sterile syringe with Milli-Q water and filtered through a $0.45 \mu \mathrm{m}$ nylon filter membrane (Whatman) was transferred to a disposable sizing cuvette (Sarsted, Germany) (DLS) or to a disposable zeta cell (Malvern, UK) ( $\zeta$-potential). The experiments were started 15 min after the sample had reached thermal equilibrium. In DLS, each sample was measured 15 times and each measurement consisted of 13 to 45 acquisitions. In $\zeta$-potential, each sample was measured 25 times and each measurement consisted of 65 acquisitions. Repeated measurements allowed error estimation.

Using the Malvern software package, on the DLS experiments the autocorrelation function of the scattered intensity was analyzed by means of the inverse Laplace transformation program CONTIN, and the hydrodynamic diameter, $d(\mathrm{H})$, was calculated using the Stokes-Einstein equation:

$$
d(\mathrm{H})=\frac{k \mathrm{~T}}{3 \pi \eta D}
$$

where $d(\mathrm{H})$ describes the hydrodynamic diameter, $D$ denotes the translational diffusion coefficient, $k$ is the Boltzman constant, $T$ is the absolute temperature, and $\eta$ is the viscosity.

The $\zeta$-potential was calculated by the Malvern software package using Henry's equation that relates electrophoretic mobility to $\zeta$-potential

$$
U_{\mathrm{E}}=\frac{2 \varepsilon \zeta}{3 \eta} f(\kappa R)
$$

where $U_{\mathrm{E}}$ is the electrophoretic mobility, $\varepsilon$ and $\eta$ are the dielectric constant and viscosity of the solvent, respectively, and $\zeta$ is the $\zeta$-potential. Henry's function, $f(\kappa \mathrm{R})$, was approximated by the Smoluchowski approximation, $f(\kappa \mathrm{R})=1.5$, because the $\zeta$-potential measurements were performed in aqueous solution.

Data obtained for different [Cyt c]/[AuNP] ratios was fitted to a Langmuir adsorption isotherm (eq 3) using Origin 7.5 Pro software.

\section{Results}

Characterization of the As-Prepared AuNPs. The SPR band of the as-prepared AuNPs appeared at $520 \mathrm{~nm}$, as determined by UV-visible spectroscopy, and no changes were observed upon $\mathrm{pH}$ variation of the solution in the range 4-11. AFM measurements of the as prepared AuNPs revealed an average nanoparticle diameter of $11.5 \mathrm{~nm}$ (inset of Figure 2), 


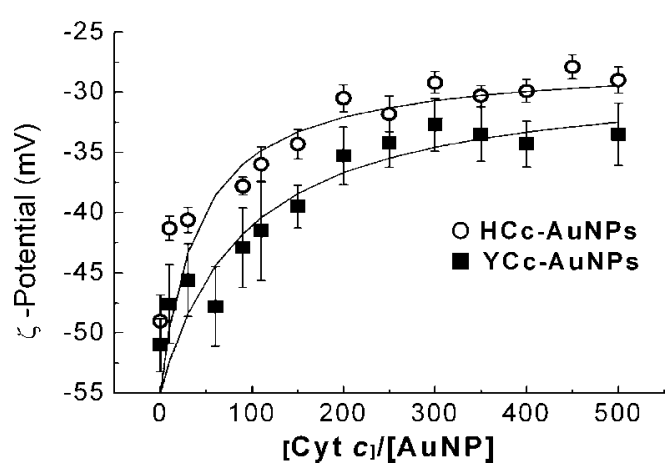

Figure 1. $\zeta$-Potential of each bionanoconjugates determined as a function of the $[\mathrm{Cyt} c] /[\mathrm{AuNP}]$ ratio at $\mathrm{pH} 11$. Each point is an average of triplicate experiments. Black lines represent fits to Langmuir adsorption isotherms (eq 3). Fitting parameters are presented in Table 1.

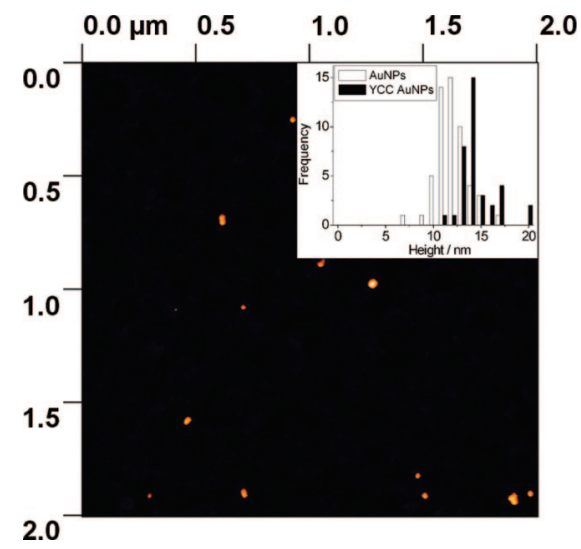

Figure 2. Tapping mode AFM image of $\mathrm{YCc}-\mathrm{AuNP}$ bionanoconjugates. The $\mathrm{z}$ (height) range is $19.3 \mathrm{~nm}$. Inset: Histogram comparing heights for as prepared AuNP (white bars) and YCc-AuNP bionanoconjugates (black bars).

in agreement with the value determined by transmission electron microscopy (TEM) for a AuNP batch prepared under the same conditions (data not shown). Determination of the hydrodynamic diameter of the AuNPs by dynamic light scattering (DLS) leads to a consistent value of $11.7 \pm 1.6 \mathrm{~nm}$.

Zeta-Potential for Evaluation of the Bionanoconjugates Surface Coverage. In obtaining proof of the formation of the Cyt $c$-AuNP bionanoconjugates, measurement of the $\zeta$-potential of the nanoparticles obtained with different Cyt $c$ concentrations was instrumental, as this technique measures the electrical potential at the surface of the bionanoconjugates. The magnitude of this surface potential is in turn related to the surface charge and the thickness of the double layer, and so it depends on the amount of protein (considered as a charged polymer) adsorbed at the bionanoconjugate surface. It is expected that as this amount increases, the surface charge will vary until it attains a stable value, indicative that no more protein is adsorbing to the bionanoconjugate. Such a correlation was previously found for bovine serum albumin and lysozyme adsorption to ceramic particles $^{45}$ and also was utilized to determine quantum dot surface coverage with $\mathrm{P} 450$ enzyme. ${ }^{11}$ In order to measure $\zeta$-potential values in conditions where bionanoconjugates are well dispersed in solution and no aggregation is present, measurements were made at $\mathrm{pH} 11$ for bare AuNPs and for increasing concentrations of both cytochromes incubated with the same concentration of AuNP, that is, increasing [Cyt $c] /\left[\right.$ AuNP] ratios (Figure 1) at a constant temperature of $25^{\circ} \mathrm{C}$. AuNP alone in solution presented a $\zeta$-potential of $-49.0 \pm 4.2$
TABLE 1: Langmuir Adsorption Isotherm Fitting Parameters for Equation 3 Describing $\zeta$-Potential Dependence $(\mathrm{mV})$ on the $[\mathrm{Cyt} \mathrm{c}] /[\mathrm{AuNP}]$ Ratio for Both Bionanoconjugates

\begin{tabular}{cccc}
\hline & $\xi \operatorname{pot}_{\max }(\mathrm{mV})$ & $K_{\mathrm{L}}$ & $\zeta \operatorname{pot}_{\max } \cdot K_{\mathrm{L}}(\mathrm{mV})$ \\
\hline $\mathrm{HCc}-$ AuNP & $-27.6 \pm 2.2$ & $0.025 \pm 0.010$ & -0.68 \\
$\mathrm{YCc}-$ AuNP & $-28.4 \pm 2.6$ & $0.011 \pm 0.004$ & -0.29
\end{tabular}

$\mathrm{mV}$, a value that agrees with previous work on citrate-stabilized AuNPs. ${ }^{4}$ For $\mathrm{HCc}-$ AuNPs, the $\zeta$-potential increases to ca. -30 $\mathrm{mV}$ as the $[\mathrm{HCc}] /[\mathrm{AuNP}]$ ratio increases to 200, keeping approximately that same value for higher $[\mathrm{HCc}] /[\mathrm{AuNP}]$ ratios. $\mathrm{HCc}$ alone presents a $\zeta$-potential of $-14.3 \pm 1.6 \mathrm{mV}$ at two different protein concentrations $(1.5$ and $10 \mu \mathrm{M})$. The fact that $\mathrm{HCc}-\mathrm{AuNPs}$ bionanoconjugates present a $\zeta$-potential that is ca. $20 \mathrm{mV}$ less negative than for AuNPs alone seems to indicate that HCc imparts some of its more positive surface charge characteristics to the AuNPs. The same is true for the $\mathrm{YCc}-\mathrm{AuNPs}$ bionanoconjugates that shifted the $\xi$-potential for AuNPs alone from -49 to $-35 \mathrm{mV}$ for a $[\mathrm{YCc}] /[\mathrm{AuNP}]$ ratio of 200 with YCc alone presenting a $\zeta$-potential close to $0 \mathrm{mV}$ $(-0.30 \pm 1.4 \mathrm{mV})$. The fact that both proteins alone present $\zeta$-potential that is close to zero should not be surprising as measurements were taken at $\mathrm{pH} 11$, close to the isoelectric point (pI) of both proteins ( $\mathrm{pI}=9.6$ for $\mathrm{HCc}$ and $\mathrm{pI}=9.5$ for $\mathrm{YCc}$, as determined by the "Compute $\mathrm{pI} / \mathrm{Mw}$ " tool of ExPASy ${ }^{46}$ ).

In studies of protein adsorption to gold surfaces at constant temperature, a Langmuir isotherm can be fitted to frequency changes of a gold-quartz crystal as a function of protein concentration. ${ }^{4,12}$ These results for a gold surface encouraged us to do a parallel between the frequency changes and the $\zeta$-potential, as both can be indirect measures of protein adsorption. In fact, $\zeta$-potential is related to the surface charge of the portion of the adsorbed protein molecule that is exposed to the solvent. As more protein is adsorbed at the AuNP surface, the formed bionanoconjugate increases its surface potential away from the very negative value observed for the citrate-stabilized AuNP, until a plateau is attained corresponding to homogeneous protein coverage, as observed in Figure 1. This approach led to a Langmuir adsorption isotherm type equation (eq 3 ):

$$
\xi \text { pot }=\frac{\xi \operatorname{pot}_{\max } K_{\mathrm{L}} R}{1+K_{\mathrm{L}} R}
$$

in which $\xi$ pot is the $\zeta$-potential measured for a bionanoconjugate with a surface coverage $R=[$ Cyt $c] /[\mathrm{AuNP}], \zeta \operatorname{pot}_{\max }$ is the maximum value for $\zeta$-potential as $R$ increases and $K_{\mathrm{L}}$ is a binding constant correspondent to the value of the inverse of the concentrations ratio, $1 / R$, for one-half of $\xi$ pot $_{\max }$. According to Langmuir isotherm theory, $\zeta \operatorname{pot}_{\max } \cdot K_{\mathrm{L}}$ is related to the enthalpy changes for the adsorption process. Table 1 summarizes the parameters obtained for the fittings of the data presented in Figure 1 using eq 3.

Although DLS seemed an attractive technique to determine the hydrodynamic diameters of bionanoconjugates and evaluate protein coverage of the AuNPs, aggregation was detected even at very low $[\mathrm{Cyt} c] /[\mathrm{AuNP}]$ ratios. Independently of the method used for the data treatment and to isolate the contribution of the large aggregates from the size distribution histograms, their extensive contribution for the scattering signal prevented the utilization of data obtained by this technique. Aggregation seems to be an important factor for $\mathrm{YCc}-\mathrm{AuNP}$, even at low ratios, while $\mathrm{HCc}-\mathrm{AuNP}$, even at ratios as high as 60 , still exhibits hydrodynamic radii that, within error, are similar to the value for bare AuNPs. 
The effective number of protein molecules present in the protein layer can be calculated assuming a maximum packing efficiency for Cyt $c$ at the surface of the AuNP with face centered cubic unit cells. The protein has a hydrodynamic diameter of $2.5 \pm 0.2 \mathrm{~nm}$ ( $\mathrm{HCc}$ ), or $2.7 \pm 0.2 \mathrm{~nm}$ (YCc) as determined by DLS. The crystallographic dimensions of the protein were previously determined as $3.0 \mathrm{~nm} \times 3.4 \mathrm{~nm} \times 3.4$ $\mathrm{nm},{ }^{12}$ but we decided to use the average solution diameter as determined by DLS due to the experimental conditions used for those measurements being closer to the ones present in the formation of the bionanoconjugates. The hydrodynamic diameter of the AuNPs alone was determined by DLS as $11.7 \pm 1.6 \mathrm{~nm}$. This computes to 72 cytochrome $c$ molecules adsorbed to the surface of the AuNP. A second protein layer on top of this one would include 145 protein molecules, thus both monolayers would have to be considered (217 protein molecules in two adjacent monolayers) in order to explain the stabilization of the $\zeta$-potential values at around 200 protein molecules per AuNP. As one of Langmuir isotherm assumptions is that adsorption does not proceed beyond monolayer coverage, the calculated two monolayers are probably not a reasonable approach to our system and an alternate, dynamic model is proposed in the Discussion below. For subsequent work, we produced bionanoconjugates with $[\mathrm{Cyt} c] /[\mathrm{AuNP}]=250$ to guarantee a fully stable surface charge corresponding to complete protein coverage in the bionanoconjugates. Other authors have previously used a similar ratio, based on different experimental evidence. ${ }^{6,7}$

Characterization of the Bionanoconjugates by AFM. AFM was applied to both bionanoconjugates in dried samples. Results for the $\mathrm{YCc}-\mathrm{AuNP}$ bionanoconjugates revealed individual structures with an average height of $13.9 \mathrm{~nm}$ (Figure 2), agreeing with an increase of ca. $2.4 \mathrm{~nm}$ in relation to AuNPs alone (average $11.5 \mathrm{~nm}$; inset in Figure 2). The consistent detection in this sample of these $14 \mathrm{~nm}$ height structures seems to indicate that YCc binds to the AuNPs in a manner that resists sample drying, forming a protein layer with an average radius of $2 \mathrm{~nm}$, consistent with monolayer coverage. In contrast, for HCc-AuNP very few particles greater than $10 \mathrm{~nm}$ could be detected, but instead the majority of the sample was made up of smaller particles with heights corresponding to mean diameters of 3.0 \pm 0.4 . The small structures most probably correspond to $\mathrm{HCc}$ alone that came out of contact with the AuNP, as the hydrodynamic diameter of HCc was determined by DLS to be $2.5 \pm 0.2 \mathrm{~nm}$. This data seems to indicate that HCc-AuNP bionanoconjugates are unstable under AFM measurements conditions, and probably sample drying is enough to separate both constituents. We assume that the AuNPs without the HCc were unstable and aggregated.

Bionanoconjugate pH Behavior As Determined by UV-vis Spectroscopy. Both AuNP-HCc and AuNP-YCc bionanoconjugates as prepared presented a plasmon resonance band at $525 \mathrm{~nm}$, a $5 \mathrm{~nm}$ red-shift in relation to the as-prepared AuNPs. This SPR shift corresponds to alterations of the refractive index at the AuNP and constitutes further evidence for the presence of a protein layer. Figure 3 presents UV-vis spectra as a function of $\mathrm{pH}$ for both bionanoconjugates. As the $\mathrm{pH}$ lowered from 11.0 to 4.0 , the $\mathrm{YCc}-\mathrm{AuNP}$ plasmon band was practically unaltered down to $\mathrm{pH} 9$ but red-shifted abruptly to $551 \mathrm{~nm}$ at $\mathrm{pH} 8.8$ and further to $570 \mathrm{~nm}$ at $\mathrm{pH} 7.0$, with no further shifting down to $\mathrm{pH} 4$. This plasmon band shift was visually detected by a change in the color of the solution from red to blue as depicted in Figure 3, corresponding to $\mathrm{YCc}-\mathrm{AuNP}$ aggregation in solution. After $2-3 \mathrm{~h}$, the blue aggregated solutions precipitated to the bottom of the assay vials. A similar behavior
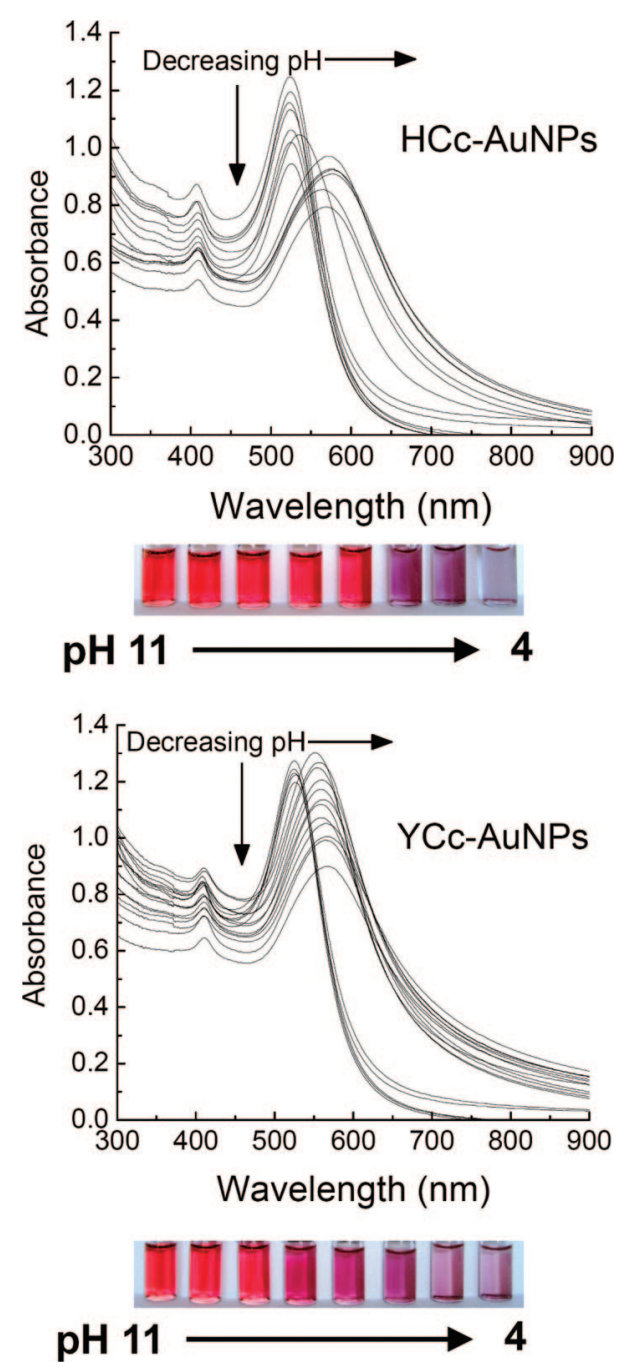

Figure 3. Absorption spectra for both bionanoconjugates as a function of the $\mathrm{pH}$ and visual effect on the solutions.

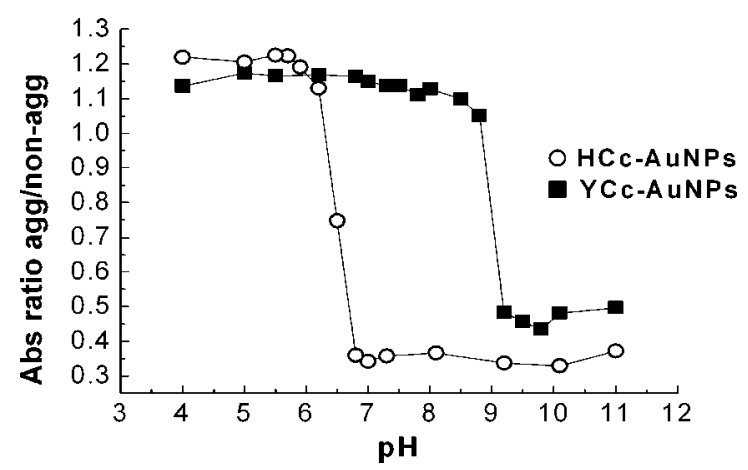

Figure 4. Variation of the ratio of absorbance for the plasmon resonance in the aggregated and nonaggregated forms, as a function of pH. Ratios for YCc-AuNP are $\left(A_{570} \mathrm{~nm} / A_{525} \mathrm{~nm}\right)$, and for $\mathrm{HCc}-\mathrm{AuNP}$ are $\left(A_{579 \mathrm{~nm}} / A_{525 \mathrm{~nm}}\right)$.

was observed upon $\mathrm{pH}$ variation of the $\mathrm{HCc}-\mathrm{AuNP}$ solutions. Relative to YCc-AuNP, however, aggregation occurred at a lower $\mathrm{pH}$ and the position of the plasmon resonance for the aggregated form was further red-shifted. Upon lowering the $\mathrm{pH}$ from 11, the $\mathrm{HCc}-\mathrm{AuNP}$ plasmon band did not change down to $\mathrm{pH} 6.8$ but red-shifted abruptly to $535 \mathrm{~nm}$ at $\mathrm{pH} 6.6$ and further to $579 \mathrm{~nm}$ at $\mathrm{pH}$ 5.0. Figure 4 presents a graphical representation of the absorbance ratios between the aggregated species (absorbance maxima at 570 and $579 \mathrm{~nm}$ for YCc-AuNP and for $\mathrm{HCc}-\mathrm{AuNP}$, respectively) and the $525 \mathrm{~nm}$ nonaggre- 
gated species as a function of the $\mathrm{pH}$. The derivative of such a representation allows the determination of a "half-titration point" for the aggregation process, evaluated to occur at $\mathrm{pH} 8.8$ for $\mathrm{YCc}-\mathrm{AuNP}$ and $\mathrm{pH} 6.2$ for $\mathrm{HCc}-\mathrm{AuNP}$. This $\mathrm{pH}$-dependent aggregation behavior of the bionanoconjugates contrasts with AuNPs alone in solution, which presented a plasmon resonance band centered at $520 \mathrm{~nm}$ for all $\mathrm{pH}$ values tested from 11.0 to 4.0. These colloidal solutions of AuNPs kept at the different $\mathrm{pH}$ values for several days presented no observable color change or plasmon resonance shift.

It has long been known that cytochromes $c$ undergo structural changes when the $\mathrm{pH}$ of the solution is varied; namely a $\mathrm{pH}$ titration followed by UV-vis spectroscopy revealed 4 different $\mathrm{p} K_{\mathrm{a}}{ }^{\prime} \mathrm{s} .{ }^{47}$ These different forms of the protein are now known to derive from altered protein folding and heme iron axial coordination. ${ }^{28}$ In the bionanoconjugates, the Soret visible band is detected at ca. $410 \mathrm{~nm}$. This band originates from the heme cofactor and although much less intense than the plasmon resonance originating from the AuNPs, a slight red-shift can be observed as an alkaline $\mathrm{pH}$ decreases. For AuNP-HCc, the Soret band shifts from $406 \mathrm{~nm}$ at $\mathrm{pH} 10.2$ to $409 \mathrm{~nm}$ at $\mathrm{pH} 8.2$, stabilizing for lower $\mathrm{pH}$ values. For AuNP-YCc, the shift is

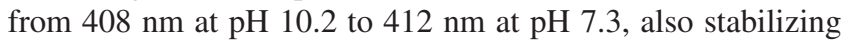
for lower $\mathrm{pH}$ values. A very similar Soret band shifting trend was obtained for the proteins alone (data not shown), as expected for the "alkaline transition" that is described to occur at $\mathrm{pH} 9.7$ for $\mathrm{YCc}$ and $\mathrm{pH} 8.3$ for $\mathrm{HCc}^{28}$ This alkaline transition corresponds to an alteration on the axial ligand of the heme group, where Met80 is replaced by a Lys residue. ${ }^{48}$ The observation of such an alkaline transition in the bionanoconjugates with spectroscopic and $\mathrm{pH}$ characteristics similar to the proteins alone, supports that the integrity of the heme pocket is most probably not affected upon protein binding to the AuNPs.

Protein Structural Changes in the Bionanoconjugates. Circular dichroism (CD) spectroscopy in the far-UV region is commonly used for determination of protein secondary structures in solution. Here it was used to evaluate the extent of the conformational changes incurred by the cytochromes $c$ in the bionanoconjugates in comparison to the proteins alone in solution, allowing an assessment of the interaction of the protein with the AuNPs. Figure 5 exhibits the far-UV CD spectra for the cytochromes $c$ alone in solution at $\mathrm{pH} 10$ and in the presence of the AuNP in the form of bionanoconjugates for high $(\mathrm{pH}$ 10) and low ( $\mathrm{pH} 4) \mathrm{pH}$ values corresponding to nonaggregation and aggregation conditions, respectively. In the case of $\mathrm{HCc}-\mathrm{AuNP}$, no appreciable variations are observed between the three situations, revealing that neither the interaction with AuNPs, nor the bionanoconjugate $\mathrm{pH}$-induced aggregation, can induce significant changes in the secondary structure of $\mathrm{HCc}$ (upper panel in Figure 5). CD spectra exhibited the two negative minima at 208 and $222 \mathrm{~nm}$ that are characteristic of HCc high $\alpha$-helix content. In contrast, YCc suffers significant alterations on its secondary structure both by interaction with AuNP and upon $\mathrm{pH}$-induced aggregation of the respective bionanoconjugates (lower panel in Figure 5). Deconvolution of the spectra using a CDSSTR algorithm (see Experimental Section) indicates that YCc in solution at high $\mathrm{pH}(\mathrm{pH} 10)$ presents mostly $\alpha$-helix conformation with a content of $31 \%$, whereas $\beta$-sheet accounts for $14 \%$ of its secondary structure. When the protein is in the bionanoconjugates, at $\mathrm{pH} 10$ its $\beta$-sheet content does not change appreciably in relation to the protein alone, whereas there is a decrease to $22 \%$ on the $\alpha$-helix content and an increase in random coil conformation. When aggregation occurs at $\mathrm{pH} 4$, there is a significant decrease of the CD signal, consistent with

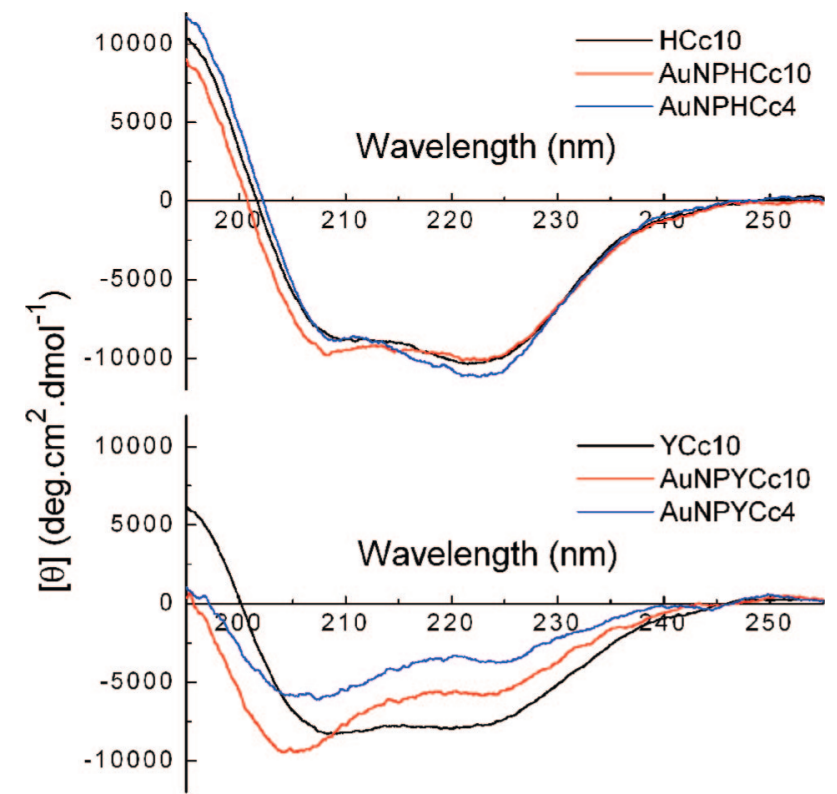

Figure 5. Far-UV CD spectra for cytochromes $c$ alone at $\mathrm{pH} 10$ (black traces), in the bionanoconjugates at $\mathrm{pH} 10$ (red traces), or at $\mathrm{pH} 4$ where aggregation occurs (blue traces). The top spectra correspond to $\mathrm{HCc}$ and $\mathrm{HCc}-\mathrm{AuNP}$ and the bottom spectra are for $\mathrm{YCc}$ and $\mathrm{YCc}-\mathrm{AuNP}$.

some extent of protein unfolding. The YCc $\alpha$-helix conformation decreases by a further $7 \%$, accounting for only $15 \%$ of the secondary structure, about half of what was determined for the protein alone in solution. Also in the aggregated condition, the $\beta$-sheet content of the protein increases to $22 \%$, an $8 \%$ increase in relation to YCc in solution. In summary, the observed decrease in the $\alpha$-helix content of YCc in the bionanoconjugate in relation to $\mathrm{YCc}$ in solution corresponds to loss of secondary structure, occurring at high $\mathrm{pH}$ and further decreasing upon aggregation at low $\mathrm{pH}$. The increase in $\beta$-sheet content that occurs only upon $\mathrm{YCc}-\mathrm{AuNP}$ aggregation at low $\mathrm{pH}$, is a clear indicator of protein aggregation.

\section{Discussion}

In this work, the formation of Cyt $c$-AuNP bionanoconjugates for both $\mathrm{YCc}$ and $\mathrm{HCc}$ was demonstrated and the characteristics of the protein adsorbed layer were analyzed using AFM, $\zeta$-potential determinations and $\mathrm{UV}-$ vis and CD spectroscopies. On the one hand, AFM results on both $\mathrm{HCc}$ and YCc bionanoconjugates, presented definite proof of YCc binding to AuNP by forming a tight protein monolayer that cannot be removed upon sample drying in contrast with $\mathrm{HCc}$ that probably detaches from the AuNP when the HCc bionanoconjugate is dried upon preparation of the AFM sample. On the other hand, $\zeta$-potential data demonstrated the formation of both bionanoconjugates by an observed loss of the AuNPs negative surface potential when incubated with increasing amounts of protein. The $\zeta$-potential data was acquired in solution in nonaggregated conditions ( $\mathrm{pH} \mathrm{11)} \mathrm{and} \mathrm{follows} \mathrm{an} \mathrm{adsorption} \mathrm{isotherm} \mathrm{as} \mathrm{the}$ protein concentration increases, indicative of the formation of a protein layer at the AuNP surface. As observed in a study of quantum dot surface coverage with hexahistidine-tagged P450 enzyme, ${ }^{11}$ the negative surface charge of our AuNPs became less negative upon protein adsorption. The $\zeta$-potential data for the bionanoconjugates formed with increasing protein concentration could be fitted to a Langmuir isotherm (Figure 2). It is an assumption of the Langmuir adsorption isotherm model that 
adsorption occurs via a single monolayer. So in order to explain the formation of stable bionanoconjugates with more than 200 protein molecules per AuNP, it should be considered that the protein adsorption process exceeds the jamming limit, ${ }^{12}$ implying that the proteins display significant lateral mobility that allows the formation of a denser monolayer. This mobility is possible for $\mathrm{HCc}$ that binds to the AuNP in an electrostatic manner, but for YCc the possibility for this mobility is obviously impaired by the covalent anchorage to the AuNP in the bionanoconjugates. It is then tempting to speculate that the observed considerable decrease of the YCc $\alpha$-helix content upon contact with AuNP would occur in order to allow a denser protein packing at the surface.

From the Langmuir isotherm fitting parameters obtained for both bionanoconjugates (Table 1), it is apparent that the maximum value for $\zeta$-potential is similar for both cases, whereas the $K_{\mathrm{L}}$ value for $\mathrm{HCc}$ is more than double the value for $\mathrm{YCc}$. As the product $\zeta \operatorname{pot}_{\max } \cdot K_{\mathrm{L}}$ is related to the enthalpy changes during the adsorption process, it seems that higher enthalpy changes are involved in the binding of HCc to AuNP than in the binding of the YCc to the nanoparticles. This conclusion is quite intriguing if we think that YCc binds to the AuNP via covalent bonding through Cys 102, whereas HCc relies only on electrostatic interaction for the binding process. Under $\zeta$-potential experimental conditions (nonaggregating; alkaline $\mathrm{pH})$, no secondary structure alterations occur on the proteins, as determined by $\mathrm{CD}$, so other factors like an entropic factor might be involved. As recently noted by Dawson and collaborators, ${ }^{49}$ protein-nanoparticle association may be entropy driven, being controlled by the release of water and/or counterions. Ideally, admitting that the adsorbed protein retains its native and active conformation, this release of structured water from the nanoparticle surface would constitute the driving force for protein binding to surfaces and not the energy associated with protein conformational changes.

In trying to develop a structural model for cytochrome interaction with AuNPs, we analyzed the surface electrostatic potentials of the three-dimensional (3D) X-ray structures of YCc and HCc. Figure 6 shows the surfaces that are most probably in contact with the AuNPs and the opposite surfaces, facing the solution. For HCc, the surface chosen for contacting the AuNPs is the "lysine patch" 33 face that was described as the contact domain when this protein is adsorptively bound to a negatively charged self-assembled monolayer on an electrode. This was established based on differential modification of surface amino acids to determine the electrode face and the solution face of the protein. ${ }^{50}$ The opposing face on the 3D structure was then considered to be facing the solution. For YCc, the surface chosen to interact with the AuNPs is the face presenting the Cys 102 residue, the opposite face being again in contact with the solution. From the figure, it is noticeable that for HCc the face in contact with the AuNP presents an extremely positive surface potential, the solution face being less positively charged; for YCc is it noticeable that neutral and negatively charged residues surround the AuNP-binding Cys 102, causing an electrostatically unfavorable interaction with the AuNP surface. This "forced" interaction of YCc through a covalent linkage with negatively charged AuNPs has been considered before as potentially destabilizing for the protein structure. ${ }^{9}$ This destabilization was reflected in the decrease of the $\alpha$-helix content of the protein in the bionanoconjugates under nonaggregating conditions, from 31 to $22 \%$, accompanied by an increase in random coil and subsequent structural unfolding, reflected in a $\beta$-sheet content increase upon bionanoconjugate
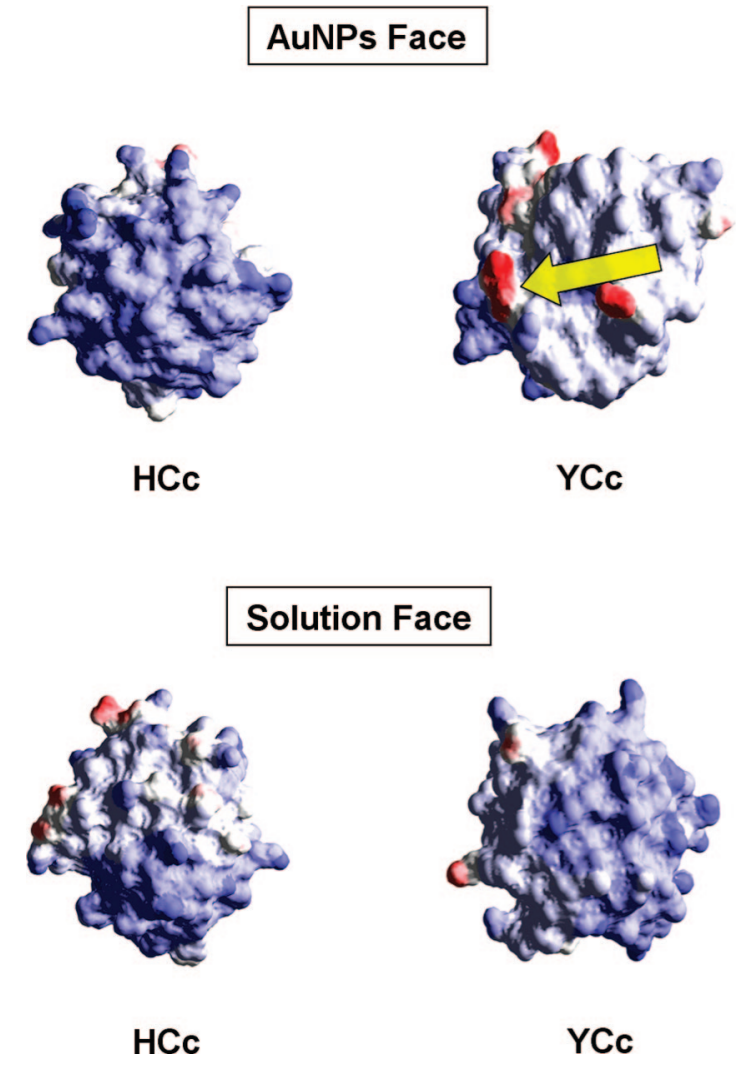

Figure 6. Surface electrostatic potentials of the 3D X-ray structures of cytochromes $c$ used in this work, exhibiting the face that is most probably in direct contact with the AuNPs (top) and the opposite face that is most probably exposed to the solution (bottom) for HCc (PDB access code 1HRC) and YCc (PDB access code 1YCC). The yellow arrow points at Cys 102 in the latter protein. Red- and blue-colored zones correspond to negative and positive potentials, respectively, for a range of potentials from -2 to $12 \mathrm{KTe}^{-1}$. Figures were prepared using Swiss-Pdb Viewer 3.7.

$\mathrm{pH}$-induced aggregation. The loss of secondary structure has been described for other proteins in AuNP bionanoconjugates..$^{8,41}$ For both proteins, the positively charged surface facing the solution can be accountable for the increase in the $\zeta$-potential value observed for bionanoconjugates with different proteincovering rates (Figure 1). Although generally positively charged, the faces that are exposed to the solution in both proteins are different, in accordance with the observed differences in the $\mathrm{pH}$-induced aggregation patterns. From Figure 4, it is clear that aggregation starts at much higher $\mathrm{pH}$ in the case of $\mathrm{YCc}-\mathrm{AuNP}$ than in $\mathrm{HCc}-\mathrm{AuNP}$, reflected in "half-titration points" at $\mathrm{pH}$ 8.8 and $\mathrm{pH} 6.2$, respectively. This observation can in turn be related to a more unstable protein structure for the $\mathrm{YCc}$ bionanoconjugate. Plasmon resonance bands of the aggregated bionanoconjugates at $\mathrm{pH} 4$ occur at 570 or $579 \mathrm{~nm}$ for $\mathrm{YCc}-\mathrm{AuNP}$ and $\mathrm{HCc}-\mathrm{AuNP}$, respectively, denoting a smaller interparticle distance in the latter aggregate. In parallel, CD results show that protein conformation changes are practically absent in the aggregated $\mathrm{HCc}-\mathrm{AuNP}$ bionanoconjugate, whereas the aggregated $\mathrm{YCc}-\mathrm{AuNP}$ shows a decrease in its $\alpha$-helix content from $22 \%$ in the nonaggregated form to $15 \%$ upon aggregation, indicating a less structured protein. Most notably, the protein $\beta$-sheet content ( $14 \%$ for YCc in solution) does not change upon protein interaction with AuNP, but increases to $22 \%$ upon $\mathrm{YCc}-\mathrm{AuNP}$ aggregation. Protein partial/total unfolding, as reflected by the increase in $\beta$-sheet content, has been described as a pathway to protein aggregation in solution. These observations imply that YCc in the bionanoconjugates undergoes 
extensive secondary structure alterations upon $\mathrm{pH}$-induced bionanoconjugate aggregation, and so confirm that the associated color change can effectively be used as a probe for protein conformational changes.,27 Regarding the structure of aggregated $\mathrm{YCc}-\mathrm{AuNP}$, it can be hypothesized that the protein is in a more open conformation in relation to its $\mathrm{HCc}-\mathrm{AuNP}$ counterpart, creating a thicker spherical crown around the AuNP. Another hypothesis is that as the $\mathrm{pH}$ is lowered, HCc detaches from the AuNP allowing for smaller interparticle distances as reflected in the positions of the SPR upon aggregation. If this hypothesis is correct, then $\mathrm{HCc}$ would still influence the aggregation in spite of being free in solution, as bare AuNP do not aggregate at any $\mathrm{pH}$ in the 11-4 range. The situation finds a parallel in the work of Rotello and co-workers, ${ }^{41}$ where the authors show that thicker protein layers at the surface of functionalized AuNPs lead to higher interparticle distances.

\section{Conclusions}

In conclusion, we examined the formation and surface characteristics of Cyt $c$-AuNP bionanoconjugates using $\mathrm{HCc}$ and YCc, two eukaryotic cytochromes $c$. Evidence for the formation of stable bionanoconjugates came from the $5 \mathrm{~nm}$ redshift observed in their SPR in relation to AuNPs alone, a protein layer of ca. $2 \mathrm{~nm}$ detected by AFM around a AuNP core of 10 $\mathrm{nm}$ in $\mathrm{YCc}$ bionanoconjugates, and a uniform surface potential at ca. 200 protein molecules per AuNP, as measured by $\zeta$-potential. The fitting of the latter data to a Langmuir adsorption isotherm lead to the conclusion that the protein-AuNP binding process has a higher enthalpy in the case of HCc than for YCc. In spite of the high biochemical and 3D structural similarities between both cytochromes $c$, the generated bionanoconjugates presented remarkably distinct surface properties. Although surface potentials are similar, the protonation patterns for both systems are different, as determined by $\mathrm{pH}$-induced aggregation: YCc bionanoconjugates present an aggregation "half-titration point" for aggregation at basic $\mathrm{pH}(8.8)$, whereas the $\mathrm{HCc}$ bionanoconjugates only start to aggregate at an acidic $\mathrm{pH}(6.2)$. This behavior contrasts with a very basic and similar isoelectric point (ca. pH 10) for proteins in solution. The protein secondary structure also suffers alterations in the bionanoconjugates, but only in the case of YCc that lowers its $\alpha$-helix content when interacting with the AuNP and increases its $\beta$-sheet content, starting to unfold upon bionanoconjugate $\mathrm{pH}$-induced aggregation. These structural alterations can have implications on YCc activity. The present comparative study of two cytochromes $c$ that share highly homologous amino acid sequences and $3 \mathrm{D}$ structures, but that generate bionanoconjugates with different surface characteristics and different secondary structures for the proteins, is an example of how protein-metal nanoparticle interactions can be advantageously used to generate bionanoconjugates with novel properties and potential applications in bioassays and biosensors.

Acknowledgment. FLAD (Luso-American Foundation) and Fundação para a Ciência e a Tecnologia, Portugal, under Projects PTDC/QUI/64484/2006 and CONC-REEQ/650/2001, and PhD fellowships SFRH/BD/18630/2004 (to I.G.) and SFRH/BD/ 23604/2005 (to L.O.) are gratefully acknowledged for financial support to this work.

\section{References and Notes}

(1) Tanaka, R.; Yuhi, T.; Nagatani, N.; Endo, T.; Kerman, K.; Takamura, Y.; Tamiya, E. Anal. Bioanal. Chem. 2006, 385, 1414-20.

\section{(2)}

(2) You, C.-C.; Verma, A.; Rotello, V. M. Soft Mat. 2006, 2, 190204

(3) Baptista, P.; Pereira, E.; Eaton, P.; Doria, G.; Miranda, A.; Gomes, I.; Quaresma, P.; Franco, R. Anal. Bioanal. Chem. 2007, 391, 943-950.

(4) Brewer, S. H.; Glomm, W. R.; Johnson, M. C.; Knag, M. K.; Franzen, S. Langmuir 2005, 21, 9303-9307.

(5) Chah, S.; Hammond, M. R.; Zare, R. N. Chem. Biol. 2005, 12, 323-328.

(6) Jiang, X.; Jiang, U. G.; Jin, Y. D.; Wang, E. K.; Dong, S. J. Biomacromolecules 2005, 6, 46-53.

(7) Keating, C. D.; Kovaleski, K. M.; Natan, M. J. J. Phys. Chem. B 1998, 102, 9404-9413.

(8) Shang, L.; Wang, Y. Z.; Jiang, J. G.; Dong, S. J. Langmuir 2007, $23,2714-2721$.

(9) Aubin-Tam, M. E.; Hamad-Schifferli, K. Langmuir 2005, 21, 12080-12084.

(10) de la Fuente, J. M.; Penades, S. Biochim. Biophys. Acta 2006, 1760, 636-51.

(11) Ipe, B. I.; Shukla, A.; Lu, H. C.; Zou, B.; Rehage, H.; Niemeyer, C. M. ChemPhysChem. 2006, 7, 1112-1118.

(12) Kaufman, E. D.; Belyea, J.; Johnson, M. C.; Nicholson, Z. M.; Ricks, J. L.; Shah, P. K.; Bayless, M.; Pettersson, T.; Feldoto, Z.; Blomberg, E.; Claesson, P.; Franzen, S. Langmuir 2007, 23, 6053-6062.

(13) You, C. C.; De, M.; Rotello, V. M. Curr. Opin. Chem. Biol. 2005, 9, 639-646.

(14) Phillips, R. L.; Miranda, O. R.; You, C. C.; Rotello, V. M.; Bunz, U. H. Angew. Chem., Int. Ed. 2008, 47, 2590-4.

(15) You, C.-C.; Miranda, O. R.; Gider, B.; Ghosh, P. S.; Kim, I.-B.; Erdogan, B.; Krovi, S. A.; Bunz, U. H. F.; Rotello, V. M. Nat. Nanotechnol. 2007, 2, 318-323.

(16) Lindman, S.; Lynch, I.; Thulin, E.; Nilsson, H.; Dawson, K. A.; Linse, S. Nano Lett. 2007, 7, 914-20.

(17) Eustis, S.; El-Sayed, M. A. Chem. Soc. Rev. 2006, 35, 209-17.

(18) Jain, P. K.; Lee, K. S.; El-Sayed, I. H.; El-Sayed, M. A. J. Phys. Chem. B 2006, 110, 7238-7248.

(19) Khlebtsov, N. G.; Trachuk, L. A.; Mel'nikov, A. G. Opt. Spectrosc. 2005, 98, 77-83.

(20) Lee, K. S.; El-Sayed, M. A. J Phys Chem B 2005, 109, 2033120338.

(21) Sonnichsen, C.; Reinhard, B. M.; Liphardt, J.; Alivisatos, A. P. Nat. Biotechnol. 2005, 23, 741-5.

(22) Baptista, P. V.; Koziol-Montewka, M.; Paluch-Oles, J.; Doria, G.; Franco, R. Clin. Chem. 2006, 52, 1433-4.

(23) Thaxton, C. S.; Georganopoulou, D. G.; Mirkin, C. A. Clin. Chim. Acta 2006, 363, 120-126.

(24) Sun, L.; Liu, D.; Wang, Z. Anal. Chem. 2007, 79, 773-777.

(25) Wang, Z.; Levy, R.; Fernig, D. G.; Brust, M. J. Am. Chem. Soc. 2006, 128, 2214-5.

(26) Xu, X. Y.; Han, M. S.; Mirkin, C. A. Angew. Chem., Int. Ed. 2007, $46,3468-3470$.

(27) Chah, S.; Kumar, C. V.; Hammond, M. R.; Zare, R. N. Anal. Chem. 2004, 76, 2112-2117.

(28) Scott, R. A.; Mauk, A. G. Cytochrome c: A multidisciplinary approach; University Science Books: Sausalito, CA, 1995.

(29) Kluck, R. M.; BossyWetzel, E.; Green, D. R.; Newmeyer, D. D. Science 1997, 275, 1132-1136.

(30) Kluck, R. M.; Martin, S. J.; Hoffman, B. M.; Zhou, J. S.; Green, D. R.; Newmeyer, D. D. EMBO J. 1997, 16, 4639-4649.

(31) Thompson, J. D.; Higgins, D. G.; Gibson, T. J. Nucleic Acids Res. 1994, 22, 4673-80.

(32) Yu, T. N.; Wang, X. D.; Purring-Koch, C.; Wei, Y.; McLendon, G. L. J. Biol. Chem. 2001, 276, 13034-13038.

(33) Banci, L.; Bertini, I.; Rosato, A.; Varani, G. J. Biol. Inorg. Chem. 1999, 4, 824-837.

(34) Rieder, R.; Bosshard, H. R. J. Biol. Chem. 1980, 255, 4732-4739.

(35) Wang, L.; Waldeck, D. H. J. Phys. Chem. C 2008, 112, 13511356

(36) Louie, G. V.; Brayer, G. D. J. Mol. Biol. 1990, 214, 527-555.

(37) Gerunda, M.; Bortolotti, C. A.; Alessandrini, A.; Sola, M.; Battistuzzi, G.; Facci, P. Langmuir 2004, 20, 8812-8816.

(38) Heering, H. A.; Wiertz, F. G. M.; Dekker, C.; de Vries, S. J. Am. Chem. Soc. 2004, 126, 11103-11112.

(39) Bortolotti, C. A.; Battistuzzi, G.; Borsari, M.; Facci, P.; Ranieri, A.; Sola, M. J. Am. Chem. Soc. 2006, 128, 5444-5451.

(40) Bonanni, B.; Alliata, D.; Bizzarri, A. R.; Cannistraro, S. ChemPhysChem. 2003, 4, 1183-1188.

(41) Srivastava, S.; Verma, A.; Frankamp, B. L.; Rotello, V. M. Adv. Mater. 2005, 17, 617-621.

(42) Baptista, P.; Doria, G.; Henriques, D.; Pereira, E.; Franco, R. J. Biotechnol. 2005, 119, 111-117.

(43) Lobley, A.; Whitmore, L.; Wallace, B. A. Bioinformatics 2002, $18,211-212$

(44) Lobley, A.; Wallace, B. A. Biophys. J. 2001, 80, 373a-373a. 
(45) Rezwan, K.; Studart, A. R.; Voros, J.; Gauckler, L. J. J. Phys. Chem. B 2005, 109, 14469-14474.

(46) Gasteiger, E.; Hoogland, C.; Gattiker, A.; Duvaud, S.; Wilkins, M. R.; Appel, R. D.; Bairoch, A. In The Proteomics Protocols Handbook; Walker, J. M.; Ed.; Humana Press: Totowa, NJ, 2005.

(47) Theorell, H.; Akesson, A. J. Am. Chem. Soc. 1941, 63, 18121818 .
(48) Assfalg, M.; Bertini, I.; Dolfi, A.; Turano, P.; Mauk, A. G.; Rosell, F. I.; Gray, H. B. J. Am. Chem. Soc. 2003, 125, 2913-2922.

(49) Dawson, K. A.; Linse, S.; Lynch, I. E-Nano Newsletter 2007, 10, 23-34.

(50) Xu, J. S.; Bowden, E. F. J. Am. Chem. Soc. 2006, 128, 6813-6822. JP804766V 